\title{
Regularized thermal lattice Boltzmann method for natural convection with large temperature differences
}

\author{
Yong-Liang Feng ${ }^{\mathrm{a}, *}$, Shao-Long Guo ${ }^{\mathrm{b}}$, Wen-Quan Tao ${ }^{\mathrm{b}}$, Pierre Sagaut ${ }^{\mathrm{a}, * *}$ \\ ${ }^{a}$ Aix Marseille Univ., CNRS, Centrale Marseille, M2P2 UMR 7340, 13451 Marseille, France \\ ${ }^{\mathrm{b}}$ Key Laboratory of Thermo-fluid Science and Engineering of MOE, School of Energy \& Power Engineering, Xian Jiaotong University, Xian 710049, China
}

\section{A R T I C L E I N F O}

\section{Keywords:}

Lattice Boltzmann method

High Rayleigh number

Large temperature difference

Perfect gas law

\begin{abstract}
A B S T R A C T
A new thermal lattice Boltzmann (LB) method is proposed for the simulation of natural convection with large temperature differences and high Rayleigh number. A regularization procedure is developed on LB equation with a third order expansion of equilibrium distribution functions, in which a temperature term is involved to recover the equation of state for perfect gas. A hybrid approach is presented to couple mass conservation equation, momentum conservation equations and temperature evolution equation. A simple and robust non-conservative form of temperature transport equation is adopted and solved by the finite volume method. A comparison study between classical Double Distribution Function (DDF) model and the hybrid finite volume model with different integration schemes is presented to demonstrate both consistency and accuracy of hybrid models. The proposed model is assessed by simulating several test cases, namely the two-dimensional non-Boussinesq natural convection in a square cavity with large horizontal temperature differences and two unsteady natural convection flows in a tall enclosure at high Rayleigh number. The present method can accurately predict both the steady and unsteady nonBoussinesq convection flows with significant heat transfer. For unsteady natural convection, oscillations with chaotic feature can be well captured in large temperature gradient conditions.
\end{abstract}

\section{Introduction}

In many natural phenomena and technological processes, density and physical properties are strongly dependent on temperature, such as natural convection, thermal flows in solar receivers, cooling air flows around internal combustion engines and many other engineering applications. In these thermal process, the density variations in the flow field are primarily induced by the heating and cooling. The density variation is due to large temperature differences, rather than to the pressure changes as in high speed flows [1]. Due to their importance for engineering applications, the thermal convection with variable density in the low Mach number limit has attracted a lot of interest over the years.

The lattice Boltzmann method (LBM) is a widely adopted approach for simulating fluid flows and complex physical phenomena $[2,3]$. Compared with the conventional computational fluid dynamics methods, the kinetic nature of LBM leads to many distinctive features, e.g. parallel computing scalability, efficiency in

\footnotetext{
* Corresponding author.

** Corresponding author.

E-mail addresses: yongliang.feng@univ-amu.fr (Y.-L. Feng), pierre.sagaut@ univ-amu.fr (P. Sagaut).
}

computing unsteady flows with high accuracy and its suitability to handle interaction between fluids and solids.

The LBM has achieved great success in simulating nearly incompressible and thermal fluid flows [4-6]. Furthermore, there has been an ongoing effort in construction of stable LB models and schemes to simulate fully compressible and weakly compressible thermal flows. An extended lattice Boltzmann model was proposed for the simulation of low Mach number flows with significant density changes [7], which is limited to open systems. Hung and Yang [8] and Li et al. [9] proposed a type of coupled double distribution function (DDF) lattice Boltzmann model for thermal flows with a complicated correction term in two-dimensional space based on a multiple relaxation time collision model. A three-dimensional DDF thermal lattice Boltzmann model with general correcting term for thermal flows with variable density was developed by Feng et al. [10].

The numerical stability of collision models is among the key issues faced when developing a LB model for thermal flows with significant density variations and strong convection. The most commonly used lattice Boltzmann collision model is the single time relaxation process referred to as the Bhatnagar-Gross-Krook (BGK) model [11]. In order to overcome the insufficient stability observed in the BGK model, several improved collision models with enhanced stability have been proposed. The multiple relax- 
ation time (MRT) model was proposed by Lallemand and Luo [12], in which the collision process is modeled in the moment space rather than in the discrete velocities space as in BGK model. The entropic lattice Boltzmann (ELB) model was developed by introducing a stabilizing process via Boltzmanns $H$ theorem [13]. Recently, both basic regularized LBGK (RLBGK) model and improved one were extended to higher order lattices both for high Reynolds number flows and for high Mach number flows [14-17]. In RLBGK method, a pre-collision operator is introduced to improve convergence properties at a very moderate computational overhead. These models were shown to provide significant improvements over the LBGK method in many high Reynolds number flows. Moreover, the regularized LBGK model appears to offer a simple and parameter-free option to save significant computational costs over the LBGK model.

The accuracy and numerical stability of hybrid thermal lattice Boltzmann method has been widely investigated in thermal flows and scalar transport process. A hybrid thermal lattice Boltzmann model was proposed by Lallemand et al. [18], where a finite difference algorithm was adopted to solve energy conservation equation. A hybrid finite difference thermal lattice model was studied in nearly incompressible convective flows [19]. Besides, a hybrid finite difference thermal model using two dimensional multiple relaxation time collision model was presented for low Mach number compressible flows [20]. As reported in studies of Li et al. [21] recently, the hybrid finite difference thermal model can simply avoid a spurious source term in thermal lattice Boltzmann models with force terms. Moreover, the robustness of hybrid approach was performed in simulation of highly compressible flows [22].

As reported in the literature, the thermal lattice Boltzmann models have been developed for thermal convection. However, robustness and accuracy of thermal LB models in simulating natural convection with strong temperature gradient at high Rayleigh numbers remains still an open question. In this paper, we aim at developing a hybrid thermal lattice Boltzmann model for natural convection with large temperature differences in the low Mach number limit. The rest of this article is organized as follows. In Section 2, both an explicit hybrid model and an implicit one are analyzed and numerically compared with a DDF thermal LB model. In Section 3, a new regularized hybrid thermal LB model with implementation of perfect gas law is derived for thermal convection flow with variable density. The validation of the present model on natural convection flows is conducted by simulating a twodimensional non-Boussinesq natural convection flow with large horizontal temperature difference and two unsteady natural convection in a tall enclosure at high Rayleigh number in Section 4. Finally, a conclusion section is given.

\section{Hybrid thermal lattice Boltzmann method}

\subsection{Isothermal lattice Boltzmann model}

The lattice Boltzmann method approximates the continuous Boltzmann equation via the discretization of both physical space and velocity space $[23,24]$. In the $D n Q m$ model, physical space in dimension $n$ is filled with a regular lattice and microscopic velocity space is discretized on a set of $m$ velocity vectors $\boldsymbol{E}=\left[\boldsymbol{c}_{0}, \ldots, \boldsymbol{c}_{i}, \ldots, \boldsymbol{c}_{m-1}\right]$. On every lattice node $\boldsymbol{x}, f_{i}(\boldsymbol{x}, t)$ denotes the density distribution of particle with velocity $\boldsymbol{c}_{i}$, thus the local density $\rho$ and momentum $\rho \boldsymbol{u}$ are defined as $\rho=\sum_{i} f_{i}$ and $\rho \boldsymbol{u}=\sum_{i} \boldsymbol{c}_{i} f_{i}$. For D2Q9 model in this study, $\boldsymbol{c}_{i}$ is given by

$$
\boldsymbol{c}_{i}= \begin{cases}(0,0) & i=0 \\ ( \pm 1,0),(0, \pm 1) & i=1-4 \\ ( \pm 1, \pm 1) & i=5-8\end{cases}
$$

The single time relaxation process (BGK) is used to model the collision term in this study.

$f_{i}(\boldsymbol{x}+\boldsymbol{c} \delta t, t+\delta t)=f_{i}(\boldsymbol{x}, t)-\frac{1}{\tau+0.5}\left[f_{i}(\boldsymbol{x}, t)-f_{i}^{e q}(\boldsymbol{x}, t)\right]$

where $\tau$ is the relaxation parameter, $\delta t$ is the time increment, which is chosen such that $\delta t=\delta \boldsymbol{x} / \boldsymbol{c} . f_{i}(x, t), f_{i}(x+\delta \boldsymbol{x}, t+\delta t)$ are the distribution functions associated with the $i$ th discrete velocity $\boldsymbol{c}_{i}$, and $f_{i}^{e q}$ is the $i$ th equilibrium distribution function, which is given by $[23,24]$.

$f_{i}^{e q}=\rho w_{i}\left[1+\frac{\boldsymbol{c}_{i} \cdot \boldsymbol{u}}{c_{s}^{2}}+\frac{\left(\boldsymbol{c}_{i} \cdot \boldsymbol{u}\right)^{2}}{2 c_{s}^{4}}-\frac{\boldsymbol{u}^{2}}{2 c_{s}^{2}}\right]$

where the weights $w_{i}=4 / 9, i=0 ; w_{i}=1 / 9, i=1,2,3,4$; $w_{i}=1 / 36, i=5,6,7,8 . c_{s}$ is the sound speed $\left(c_{s}=1 / \sqrt{3}\right)$.

Using the Chapman-Enskog multiscale technique [25], the Navier-Stokes equations at the second order of approximation can be obtained as follows:

$\frac{\partial \rho}{\partial t}+\nabla \cdot(\rho \boldsymbol{u})=0$

$\frac{\partial \rho \boldsymbol{u}}{\partial t}+\nabla \cdot(\rho \boldsymbol{u} \boldsymbol{u})=-\nabla p+\nabla \cdot\left[\rho v\left(\nabla \boldsymbol{u}+(\nabla \boldsymbol{u})^{T}\right)\right]$

where the pressure is related to the density by the equation of the state $p=\rho c_{s}^{2}$ and the kinematic viscosity $v$ has the relationship with relaxation parameter as:

$v=c_{s}^{2} \tau \delta t$

\subsection{Thermal LB models for incompressible thermal flows}

Before extending a hybrid thermal LB model to simulate natural convection flows with variable density, the accuracy and computational efficiency of different LB thermal models are comprehensively investigated in this section. Since double distribution function model and hybrid finite difference/volume thermal (HT) models are widely applied in thermal flows, two different HT models are studied and compared with a DDF model in the following.

\subsubsection{Double distribution function thermal LB model}

In order to compare a DDF thermal model with HT models, we briefly describe a simple lattice Boltzmann DDF model. The DDF thermal LB model relies on an additional distribution function, instead of the original single-particle distribution function, to describe the evolution of the temperature field [26,27]. Neglecting the viscous heat dissipation and the compression work in the studied thermal flows, the evolution equation of temperature distribution function $h$ in terms of temperature relaxation time can be expressed as follows:

$h_{i}\left(\boldsymbol{x}+\boldsymbol{c}_{i} \delta_{t}, t+\delta_{t}\right)-h_{i}(\boldsymbol{x}, t)=-\frac{1}{\tau_{h}+0.5}\left[h_{i}(\boldsymbol{x}, t)-h_{i}^{e q}(\boldsymbol{x}, t)\right]$

The equilibrium temperature distribution function is the second-order truncated Taylor or Hermite polynomials of Maxwellian distribution:

$h_{i}^{e q}=w_{i} \rho T\left[1+\frac{\boldsymbol{c}_{i} \cdot \boldsymbol{u}}{c_{s}^{2}}+\frac{\left(\boldsymbol{c}_{i} \cdot \boldsymbol{u}\right)^{2}}{2 c_{s}^{4}}-\frac{\boldsymbol{u}^{2}}{2 c_{s}^{2}}\right]$

where $T$ is the local temperature, which can be obtained from $\rho T=\Sigma_{i} h_{i}$. The temperature relaxation time $\tau_{h}$ are related with relaxation time of velocity filed $\tau$, which is tied by the following relation

$\operatorname{Pr}=\frac{\tau}{\tau_{h}}=\frac{v}{\kappa}$ 
where $\operatorname{Pr}$ is the Prandtl number and $\kappa$ is the thermal diffusivity. The DDF model is simple and well suited to thermal flows with different Prandtl numbers.

\subsubsection{Hybrid thermal LB models}

In the hybrid lattice Boltzmann method investigated in this study, the continuity equation and momentum equation are solved by the lattice Boltzmann equation, while the energy conservation equation is separately solved by a finite volume technique. The enthalpy equation is used in this study for thermal flows at zero or low Mach number, which is expressed by the following general convection-diffusion form:

$\rho c_{p} \frac{\partial T}{\partial t}+\rho c_{p} \boldsymbol{u} \cdot \nabla T=\nabla(\lambda \nabla T)+\frac{D p}{D t}+\Phi$

where $\Phi$ is the source term of the viscous heat dissipation.

Two types of time integration scheme are investigated in this study. The first one is the explicit second-order Runge-Kutta scheme $\left(\mathrm{HT}_{a}\right)$ while the second one is the Adams-Bashforth/ Crank-Nicolson scheme $\left(\mathrm{HT}_{b}\right)$. The two-step Runge-Kutta scheme is given as

$T^{n+1 / 2}=T^{n}+\frac{\delta t}{2} R H S\left(T^{n}\right)$
$T^{n+1}=T^{n}+R H S\left(T^{n+1 / 2}\right)$

For the Adams-Bashforth/Crank-Nicolson scheme, the convection term in Eq. (10) is discretized by Adams-Bashforth scheme while the diffusion term is discretized by Crank-Nicolson scheme. The resulted algebraic equations are solved by Bi-CGSTAB $[28,29]$.

$\boldsymbol{u} \cdot \nabla T=\frac{3}{2} \boldsymbol{u} \cdot \nabla T^{n}-\frac{1}{2} \boldsymbol{u} \cdot \nabla T^{n-1}$

$\nabla(\lambda \nabla T)=\frac{1}{2} \nabla\left(\lambda^{n+1} \nabla T^{n+1}\right)+\frac{1}{2} \nabla\left(\lambda^{n} \nabla T^{n}\right)$

The energy conservation equation is spatially discretized using Monotonic Upwind Scheme for Conservation Laws (MUSCL) and central difference (CD) scheme [30], where MUSCL or CD scheme is adopted for the convection term and the central difference scheme for the diffusion term.

In the following, computational efficiency of two HT models and the DDF thermal model is investigated by numerical experiments on a typical incompressible thermal flow. Natural convection in a square cavity with an aspect ratio equal to unity is investigated by both the DDF model and the hybrid models, which is widely investigated by lattice Boltzmann method [31-33]. The Prandtl number is taken equal to 0.71 . The Boussinesq assumption is adopted and the buoyancy force due to gravity works downwards. All surrounding walls are rigid and impermeable. The vertical walls located at $x=0$ and $x=H$ are retained to be isothermal but at different temperature, $T_{h}$ and $T_{c}$, respectively. The horizontal laterals are taken as adiabatic.

For the implementation of boundary conditions, the regularized boundary condition [34] with the reconstruction of nonequilibrium distribution functions is adopted on both density velocity distribution function and energy distribution function in the DDF model. In the HT models, the non-equilibrium regularized boundary condition is only adopted on density velocity distribution function while the Dirichlet boundary condition and the Neumann boundary condition in finite volume method are applied at constant temperature boundary and adiabatic boundary, respectively. At initial condition, the fluid inside cavity is assessed to obey the static solution at uniform temperature of $\left(T_{h}+T_{c}\right) / 2$, where non-equilibrium scheme for initial condition is implemented in density velocity and energy distribution function. In the hybrid thermal models, the macroscopic temperature is directly set to initial value and the treatment of initial condition for velocity field is the same with the DDF model.

Fig. 1 displays the temperature isocontours obtained by DDF, $\mathrm{HT}_{a}$ and $\mathrm{HT}_{b}$ on $100 \times 100$ grids at $\mathrm{Ra}=10^{3}, 10^{4}, 10^{5}$ and $10^{6}$, respectively. It is found that the temperature fields obtained using the different models are almost identical when the flow reaches a steady state. Especially, the temperature contours of two hybrid models are completely overlapped. There are only slight differences between results of the DDF model and the HT models at $R a$ number $10^{5}$ and $10^{6}$. These deviations can originate in the treatment of the boundary conditions. Comparison of flow field obtained by the DDF model and two HT models is reported in Fig. 2. The results on velocities are consistent with those comparison on the temperature field. It is shown that the two HT models yield almost the same velocity distribution compared with the DDF model. The deviations increase when Rayleigh number is increased from $10^{3}$ to $10^{6}$ and reached a maximum at $R a=10^{6}$. However, the maximum deviation is lower than $1 \%$.

Above all, computational efficiency of different thermal models is compared through the residual histories and CPU time cost. In Fig. 3 the velocity and temperature residual history for natural convection at $R a=10^{5}$ by the DDF model and the HT models are plotted along with $\mathrm{x}$-axis at bottom and $\mathrm{y}$-axis at left side. It can be seen from CPU time that both types of HT models exhibit faster convergence speed than the DDF model when these models are applied to simulate steady natural convection. In other words, two HT models spends less CPU time than the DDF model when those models are used to simulate unsteady natural convection developing in physical time. The hybrid thermal model with Runge-Kutta scheme exhibits 1.5 times higher computational efficiency than the DDF model. The computational efficiency of hybrid thermal model with Adams-Bashforth/Crank-Nicolson scheme is 1.3 times higher than DDF model. Besides, the random-access memory occupied by the HT models is much less than one used by the DDF model. It is should be emphasized that the explicit RK HT model is the fastest model among those thermal LB models.

Numerically, DDF model is not optimal from the computational standpoint, even though this numerical inefficiency can be improved somewhat by using some redundant degree of freedom in LB models in diffusion dominated condition [35]. Thus, the explicit HT model with the second order Runge-Kutta scheme will be adopted and developed in the following studies on natural convection with strong temperature gradients.

\section{Thermal LB model for non-Boussinesq natural convection}

\subsection{Hybrid thermal LB model for perfect gas}

The aim of this section is to extend the hybrid LB model to compressible convection flows with variable density. Considering the third order Hermite expansion of Maxwell-Boltzmann distribution with a variation of temperature, one obtains the following equilibrium distribution function in discrete Gauss-Hermite space [36].

$f_{i}^{e q}=f_{i}^{(0)}=\rho w_{i}\left[1+\frac{c_{i \alpha} u_{\alpha}}{c_{s}^{2}}+\frac{A_{\alpha \beta}^{(2)} Q_{i \alpha \beta}^{(2)}}{2 c_{s}^{4}}\right]+\frac{A_{\alpha \beta \gamma}^{(3)} Q_{i \alpha \beta \gamma}^{(3)}}{6 c_{s}^{6}}$

$A_{\alpha \beta}^{(2)}=u_{\alpha} u_{\beta}+(\theta-1) \delta_{\alpha \beta}, \quad Q_{i \alpha \beta}^{(2)}=c_{i \alpha} c_{i \beta}-c_{s}^{2} \delta_{\alpha \beta}$

$A_{\alpha \beta}^{(3)}=u_{\alpha} u_{\beta} u_{\gamma}+(\theta-1)[u \delta]_{\alpha \beta \gamma}, \quad Q_{i \alpha \beta}^{(3)}=c_{i \alpha} c_{i \beta} c_{i \gamma}-c_{s}^{2}[c \delta]_{\alpha \beta \gamma}$

where $\theta=R T / c_{s}^{2}$, and the moment of equilibrium distribution function on nearest neighbor type lattices (D2Q9, D3Q19, D3Q27) are summarized as follows: 


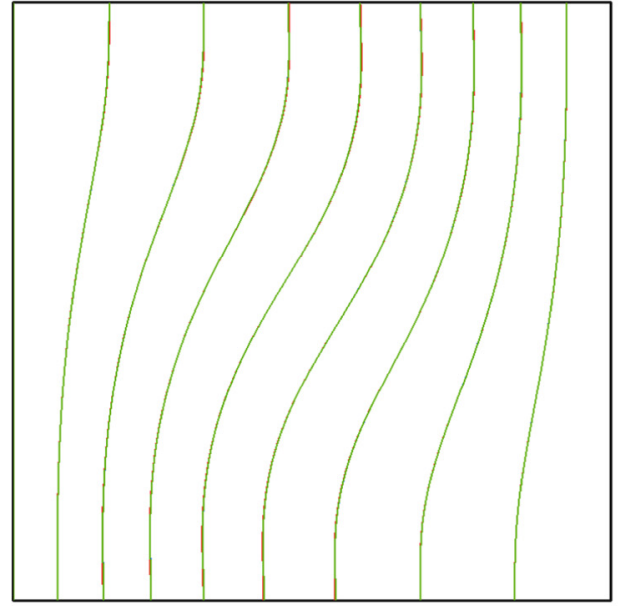

(a) $R a=10^{3}$

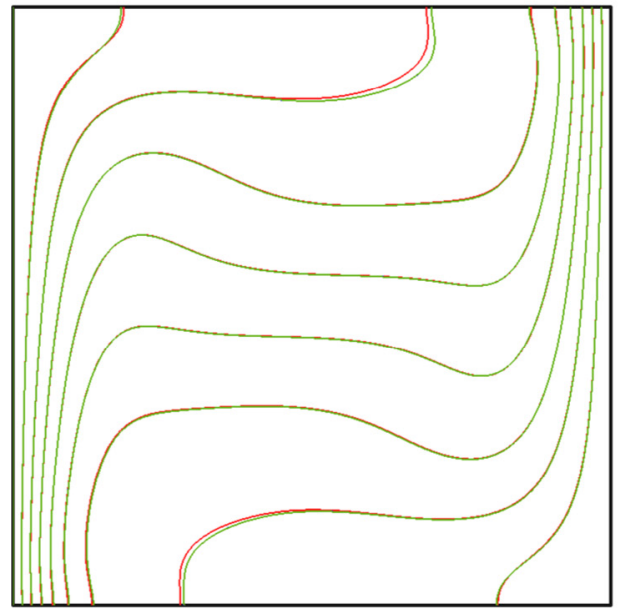

(c) $R a=10^{5}$

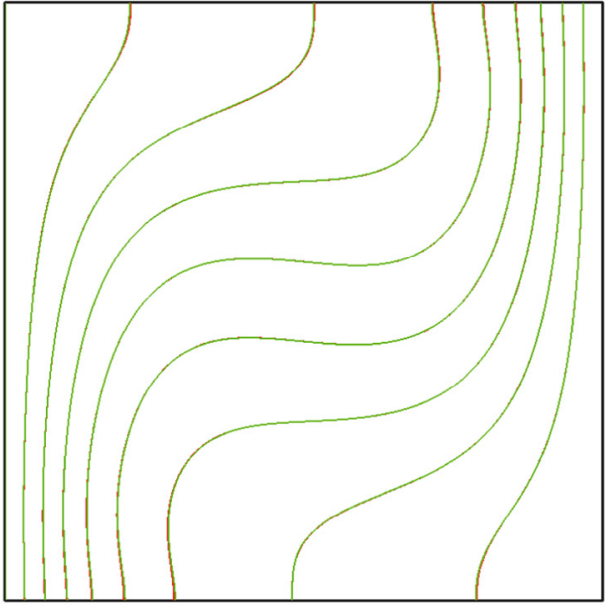

(b) $R a=10^{4}$

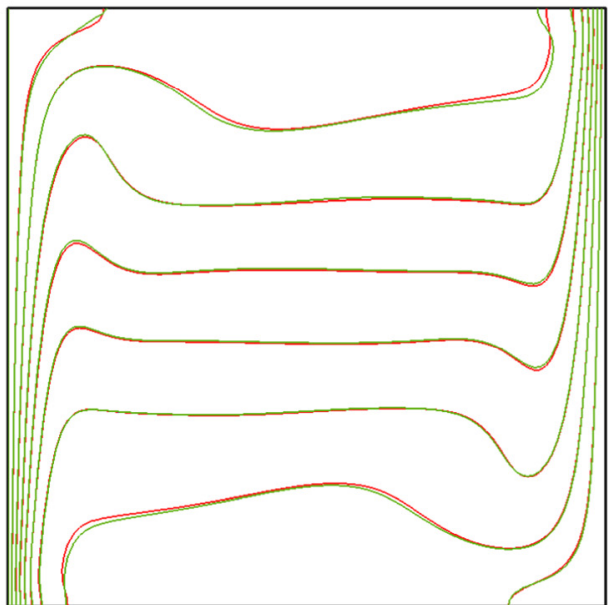

(d) $R a=10^{6}$

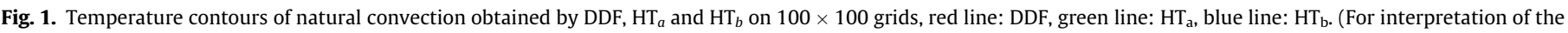
references to color in this figure legend, the reader is referred to the web version of this article.)

$\sum_{i} f_{i}^{(0)}=\rho$

$\sum_{i} f_{i}^{(0)} c_{i \alpha}=\rho u_{\alpha}$

$\sum_{i} f_{i}^{(0)} c_{i \alpha} c_{i \beta}=\rho R T \delta_{\alpha \beta}+\rho u_{\alpha} u_{\beta}$

$\sum_{i} f_{i}^{(0)} c_{i \alpha} c_{i \beta} c_{i \gamma}=\rho R T\left(u_{\alpha} \delta_{\beta \gamma}+u_{\beta} \delta_{\gamma \alpha}+u_{\gamma} \delta_{\beta \alpha}\right)+\rho u_{\alpha} u_{\beta} u_{\gamma}+\Psi_{\alpha \beta \gamma}$

where $\Psi_{\alpha \beta \gamma}$ is deviation terms due to defect of symmetry of lattices (D2Q9, D3Q19, D3Q27) on the third order moment. The detailed formula can be refer to $[9,10]$. A force term $s_{i}$ Incorporated with gravity is used to compensate the deviation and the moments of the external force term $s_{i}$ can be defined as

$\sum_{i} s_{i}=S_{0}$

$\sum_{i} s_{i} c_{i \alpha}=S_{\alpha}$ $\sum_{i} s_{i} c_{i \alpha} c_{i \beta}=S_{\alpha \beta}$

To derive macroscopic equations in the hydrodynamic limit, the density distribution function $f_{i}$ is expanded around the $f_{i}^{(0)}$ as follows:

$f_{i}=f_{i}^{(0)}+\epsilon f_{i}^{(1)}+\epsilon^{2} f_{i}^{(2)}+\cdots$

with

$\sum_{i} f_{i}^{(n)}=0, \quad \sum_{i} f_{i} c_{i}^{(n)}=0, \quad \sum_{i} f_{i}^{(n)} c_{i}^{2}=0 \quad n=1,2, \cdots$

By matching the scales of $\epsilon^{1}$ and $\epsilon^{2}$, the following equations can be obtained

$\epsilon^{1}:\left(\frac{\partial}{\partial t_{1}}+\boldsymbol{c}_{i} \cdot \nabla_{1}\right) f_{i}^{e q}+\frac{f_{i}^{(1)}}{\tau \delta_{t}}=s_{i}^{(0)}$

$\epsilon^{2}: \frac{\partial f_{i}^{e q}}{\partial t_{2}}+\left(\frac{\partial}{\partial t_{1}}+\boldsymbol{c}_{i} \cdot \nabla_{1}\right) f_{i}^{(1)}+\frac{f_{i}^{(2)}}{\tau \delta_{t}}=0$

After summing Eqs. (24) and (25) in the velocity phase space, the $t_{1}$ and $t_{2}$ order of the continuity equation and momentum equation can be derived as 


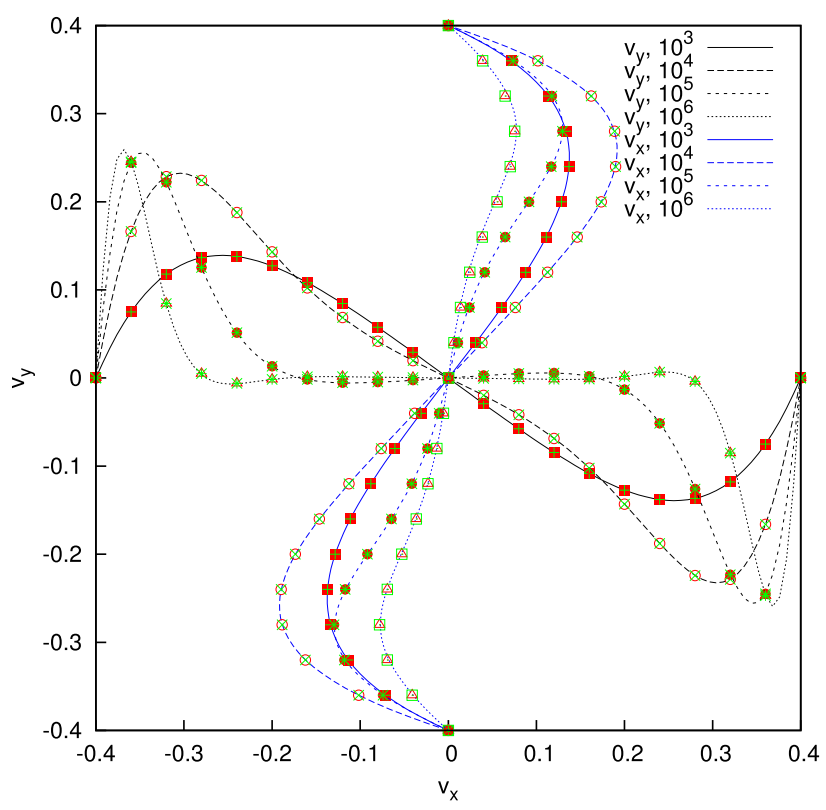

Fig. 2. Non-dimensional velocity profiles for different thermal LB models. Results are obtained by DDF (lines), $\mathrm{HT}_{a}$ (red symbols) and $\mathrm{HT}_{b}$ (green symbols) on $100 \times 100$ grids at $R a=10^{3}, 10^{4}, 10^{5}$ and $10^{6}$. Reference velocity $v_{\text {ref }}=\sqrt{R a} \mathrm{v} / \mathrm{L}$. (For interpretation of the references to color in this figure legend, the reader is referred to the web version of this article.)

$\frac{\partial \rho}{\partial t_{1}}+\frac{\partial}{\partial x_{1 \alpha}}\left(\rho u_{\alpha}\right)=S_{0}$

$\frac{\partial \rho}{\partial t_{2}}=0$

$$
\begin{gathered}
\frac{\partial}{\partial t_{1}}\left(\rho u_{\alpha}\right)+\frac{\partial}{\partial x_{1 \beta}}\left(\rho u_{\alpha} u_{\beta}+p \delta_{\alpha \beta}\right)=S_{\alpha} \\
\frac{\partial}{\partial t_{2}}\left(\rho u_{\alpha}\right)+\frac{\partial}{\partial x_{1 \beta}}\left(\sum_{i} c_{i \alpha} c_{i \beta} f_{i}^{(1)}\right)=0
\end{gathered}
$$

Rewriting $f_{i}^{(1)}$ in Eq. (29) using Eq. (26), one obtains

$$
\begin{aligned}
\sum_{i} c_{i \alpha} c_{i \beta} f_{i}^{(1)}= & -\tau \sum_{i} c_{i \alpha} c_{i \beta}\left[\left(\frac{\partial}{\partial t_{1}}+c_{i} \cdot \nabla_{1}\right) f_{i}^{e q}-s_{i}^{(0)}\right] \\
= & -\tau\left[\frac{\partial}{\partial t_{1}}\left(\rho u_{\alpha} u_{\beta}+p \delta_{\alpha \beta}\right)-S_{\alpha \beta}+\frac{\partial}{\partial x_{1 \gamma}} \Psi_{\alpha \beta \gamma}\right. \\
& \left.+\frac{\partial}{\partial x_{1 \gamma}}\left(\rho R T\left(u_{\alpha} \delta_{\beta \gamma}+u_{\beta} \delta_{\gamma \alpha}+u_{\gamma} \delta_{\beta \alpha}\right)+\rho u_{\alpha} u_{\beta} u_{\gamma}\right)\right]
\end{aligned}
$$

Taking into account the fact that derivatives of pressure scale as Mach number in the low Mach number limit, and $S_{\alpha \beta}=\frac{\partial}{\partial x_{1 \gamma}} \Psi_{\alpha \beta \gamma}$, and $S_{0}=0$, one simply obtains the following equations

$\sum_{i} c_{i \alpha} c_{i \beta} f_{i}^{(1)}=-\tau\left[p\left(\frac{\partial u_{\beta}}{\partial x_{\alpha}}+\frac{\partial u_{\alpha}}{\partial x_{\beta}}\right)+O\left(M a^{3}\right)\right]$

It can be observed that there is a deviation on bulk viscous term compared with fully compressible Navier-Stokes equations since evolution of pressure is not fully included in the energy equation adopted in this study. However, the deviation term is negligible in thermal flows at low Mach number. Collecting with the energy equation solved by hybrid finite volume approach, the final macroscopic equations are

$\frac{\partial \rho}{\partial t}+\frac{\partial}{\partial x_{\alpha}}\left(\rho u_{\alpha}\right)=0$

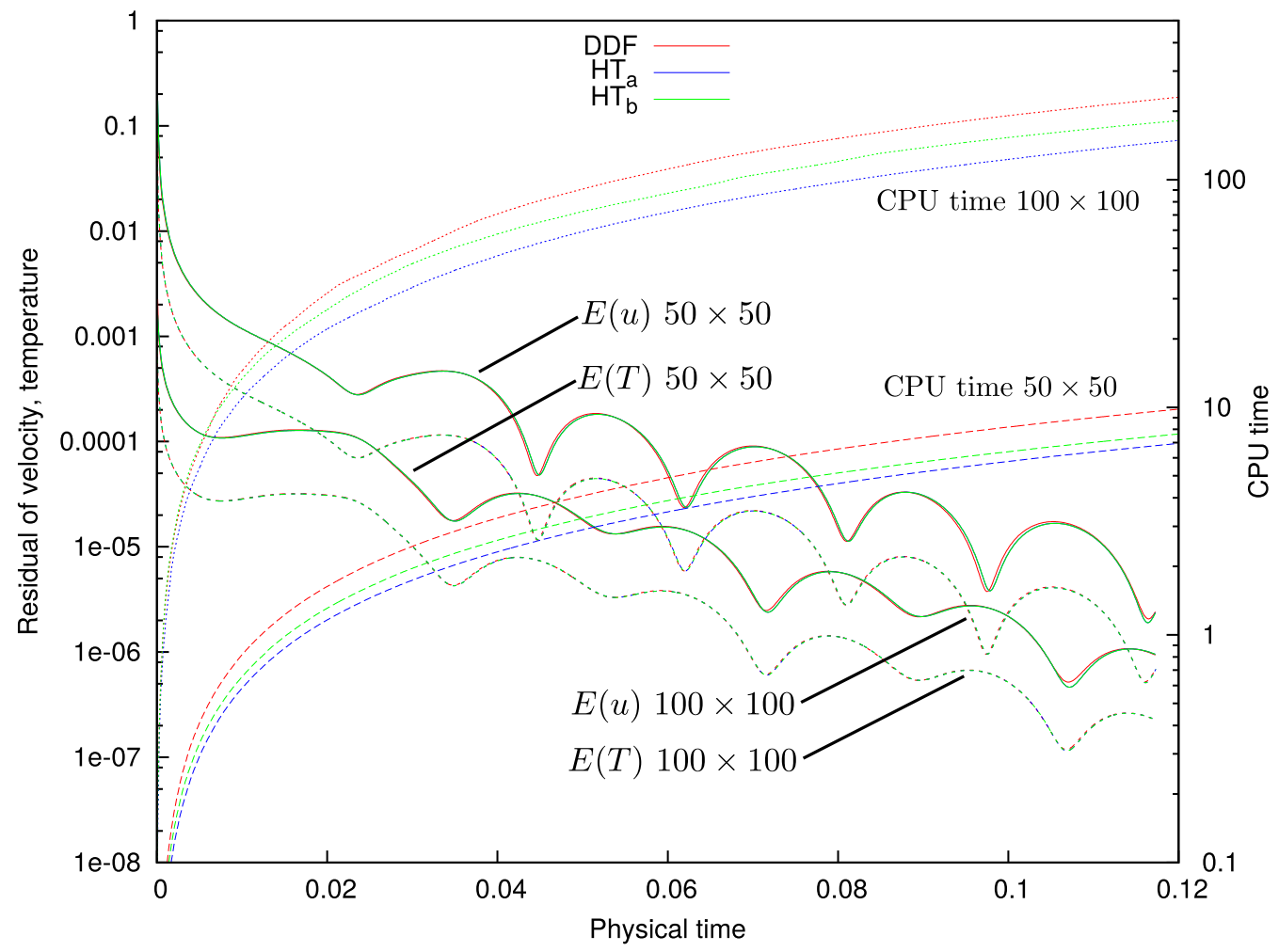

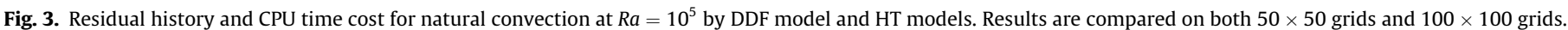

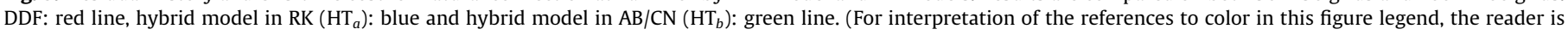
referred to the web version of this article.) 


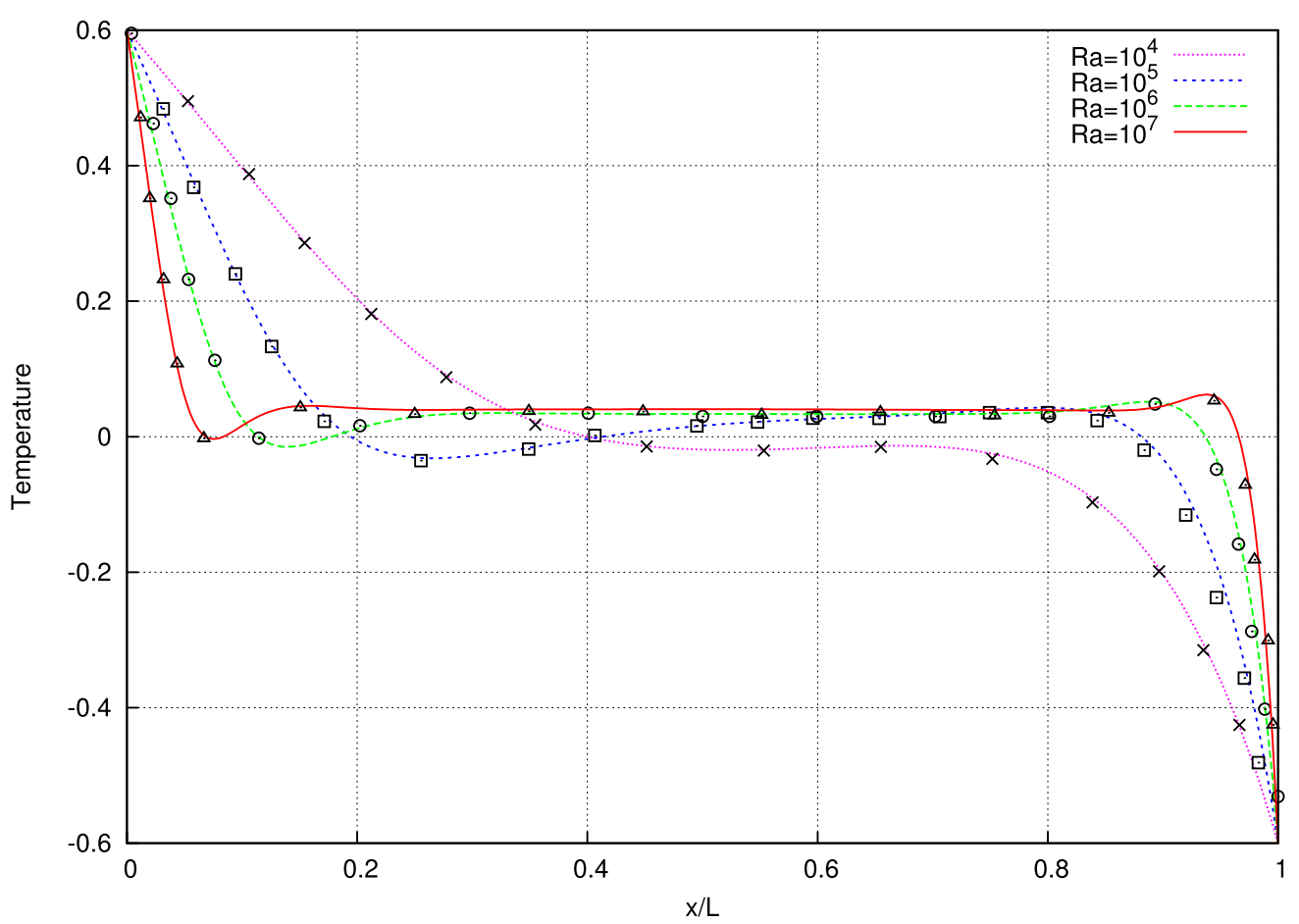

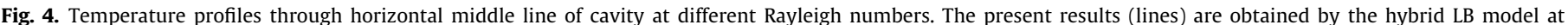
$R a=10^{4}, 10^{5}, 10^{6}$ and $10^{7}$. The symbols represent benchmark solution in [40].

$\frac{\partial}{\partial t}\left(\rho u_{\alpha}\right)+\frac{\partial}{\partial x_{\beta}}\left(\rho u_{\alpha} u_{\beta}\right)=-\frac{\partial}{\partial x_{\alpha}} p+\frac{\partial}{\partial x_{\beta}}\left[\mu\left(\frac{\partial u_{\beta}}{\partial x_{\alpha}}+\frac{\partial u_{\alpha}}{\partial x_{\beta}}\right)\right]+S_{\alpha}$

$\frac{\partial T}{\partial t}+u_{\alpha} \frac{\partial T}{\partial x_{\alpha}}=\frac{1}{\rho c_{p}} \frac{\partial}{\partial x_{\alpha}}\left(\lambda \partial x_{\alpha} T\right)+\frac{\Phi}{\rho c_{p}}$

where $p=\rho R T$ and with the dynamic viscosity $\mu=\tau p$. It is worth noting that there is a correction terms to recover the equation of state. Due to partial symmetry of nearest neighbor lattices, e.g. D2Q9 on the third moments, the correction term on implementation of perfect gas law is given in a simple form

$s_{i, c}=Q_{i x x} \frac{\partial}{\partial x}\left[\rho\left(1-\theta-u_{x}^{2}\right)\right]+Q_{i y y} \frac{\partial}{\partial y}\left[\rho\left(1-\theta-u_{y}^{2}\right)\right]$

The spatial gradients are numerically implemented using an isotropic second order central difference scheme [37]. It is noted that the Prandtl number of the present model is $\operatorname{Pr}=\mu c_{p} / \lambda$, which can be adjusted in the present hybrid thermal lattice model.

\subsection{Regularization procedure}

In order to enhance the stability of proposed model in simulating thermal flows with large temperature differences and strong convection. A regularization procedure is incorporated in the present framework of hybrid thermal LB model. The regularization procedures were proposed for improve stability of lattice Boltzmann methods [14]. They play the role of a filter on spurious ghost moments of the numerical scheme. The accuracy and stability of regularized BGK model were well analyzed in both inviscid acoustic problems [38] and turbulent flows [17]. Practically, a regularized distribution function is introduced through recomputing the non-equilibrium parts prior to the collision step.

Rewriting the lattice Boltzmann BGK equation with external force terms using the trapezoidal rule, the LBGK equation can be expressed equivalently as $\overline{f_{i}}(\boldsymbol{x}+\boldsymbol{c} \delta t, t+\delta t)=f_{i}^{e q}(\boldsymbol{x}, t)+\left(1-\frac{1}{\bar{\tau}}\right)\left[\overline{f_{i}}(\boldsymbol{x}, t)-\overline{f_{i}}{ }^{e q}(\boldsymbol{x}, t)\right]+\frac{1}{2} s_{i}(\boldsymbol{x}, t)$

${\overline{f_{i}}}^{e q}=f_{i}^{e q}-\frac{1}{2} s_{i}$

$\overline{f_{i}}=f_{i}+\frac{1}{2 \tau}\left(f_{i}-f_{i}^{e q}\right)-\frac{1}{2} s_{i}$

where $\bar{\tau}=\tau+1 / 2$, and a regularization procedure is adopted on non-equilibrium distribution $\bar{f}^{\text {neq }}={\overline{f_{i}}}_{-} \bar{f}_{i}{ }^{\text {eq }}$ to suppress the nonhydrodynamic ghost moment:

$\Pi_{\alpha \beta}^{(1)}=\sum_{i} \bar{f}_{i}^{n e q} c_{i \alpha} c_{i \beta}$,

$\mathcal{R}\left({\overline{f_{i}}}^{n e q}\right) \approx \frac{w_{i}}{2 c_{s}^{4}}\left(c_{i \alpha} c_{i \beta}-c_{s}^{2} \delta_{\alpha \beta}\right) \Pi_{\alpha \beta}^{(1)}$,

$\overline{f_{i}}(\boldsymbol{x}+\boldsymbol{c} \delta t, t+\delta t)=f_{i}^{e q}(\boldsymbol{x}, t)+\left(1-\frac{1}{\bar{\tau}}\right) \mathcal{R}\left({\overline{f_{i}}}^{\text {neq }}\right)+\frac{1}{2} s_{i}(\boldsymbol{x}, t)$

In this study, the force term $s_{i}$ consists of correction term $s_{i, c}$ and gravity term $s_{i, g}$, which is given by

$s_{i}=s_{i, c}+s_{i, g}$

$s_{i, g}=w_{i}\left[\frac{c_{i \alpha} F_{\alpha}}{c_{s}^{2}}+\frac{\left(c_{i \alpha} c_{i \beta}-c_{s}^{2} \delta_{\alpha \beta}\right) F_{\alpha \beta}}{2 c_{s}^{4}}\right]$

where $F_{\alpha}=\rho g_{\alpha}$ and $F_{\alpha \beta}=u_{\alpha} F_{\beta}+u_{\beta} F_{\alpha}$. The correction term $s_{i, c}$ is calculated from Eq. (35).

\section{Results and discussion}

First, a two-dimensional non-Boussinesq natural convection in square cavity is studied by the proposed method in different Rayleigh numbers from $10^{4}$ to $10^{7}$. Next, two unsteady natural 
convection with strong temperature gradients in a tall enclose is analyzed and compared with benchmark solutions.

\subsection{Non-Boussinesq natural convection in a square cavity}

A non-Boussinesq natural convection is investigated by the proposed thermal model and to assess the capability of the hybrid thermal model for thermal convection with significant heat transfer. In the well known Boussinesq approximation it is assumed that all fluid properties (density, viscosity, thermal diffusivity) can be considered as constant except the density $\rho$ in the body force term, where it is assumed to be a linear function of the temperature $\rho=\rho_{0}\left[1-\beta\left(T-T_{0}\right)\right]$, with $\rho_{0}$ and $T_{0}$ the reference fluid density and temperature, respectively, and $\beta$ the coefficient of thermal expansion. In the following study of natural convection, the Boussinesq approximation is not appropriate, since temperature difference is large and flow field and temperature field are fully coupled through an equation of state for perfect gas.

The left vertical wall located at $x=0$ at a high temperature $T_{h}=960 \mathrm{~K}$ and the right vertical wall located at $x=H$ at a low temperature $T_{c}=240 \mathrm{~K}$, respectively. The horizontal laterals are taken as adiabatic. The boundary conditions and initial condition are implemented as the same with setting in the Boussinesq natural convection in Section 2.

In this case, variations of thermal physical properties dependent on temperature have been taken into account in both lattice
Boltzmann equation and finite volume equation. The dynamic viscosity and thermal conductivity are defined by Sutherland law [39]:

$\frac{\mu(T)}{\mu^{*}}=\left(\frac{T}{T^{*}}\right)^{3 / 2} \frac{T^{*}+S}{T+S}$

$\lambda(T)=\frac{c_{p} \mu(T)}{\operatorname{Pr}}$

where $T^{*}=273 \mathrm{~K}, S=110.5 \mathrm{~K}$ and $\mu^{*}=1.68 \times 10^{-5} \mathrm{~kg} / \mathrm{m} / \mathrm{s}$ for air. The temperature-dependent viscosity and conductivity are realized by the variable relaxation parameter $\tau=\mu(T) / p$ with a fixed $\operatorname{Pr}$. The Rayleigh number is defined as:

$R a=\operatorname{Pr} \frac{g \rho_{0}^{2}\left(T_{h}-T_{c}\right) L^{3}}{T_{0} \mu_{0}^{2}}$

where $\operatorname{Pr}=0.71$, the reference temperature $T_{0}=\left(T_{h}+T_{c}\right) / 2, \rho_{0}$ and $\mu_{0}$ are the reference density and the reference dynamic viscosity at reference temperature, respectively.

The simulations based on the present hybrid LB model are conducted considering four Rayleigh numbers $\left(R a=10^{4}, 10^{5}, 10^{6}\right.$ and $10^{7}$ ) on grid resolution ranging from $50 \times 50$ to $400 \times 400$. Besides, a simulation on a very fine grid with $800 \times 800$ is carried at Rayleigh number $R a=10^{7}$. Fig. 4 displays the temperature profiles through horizontal middle line of cavity at the four Rayleigh

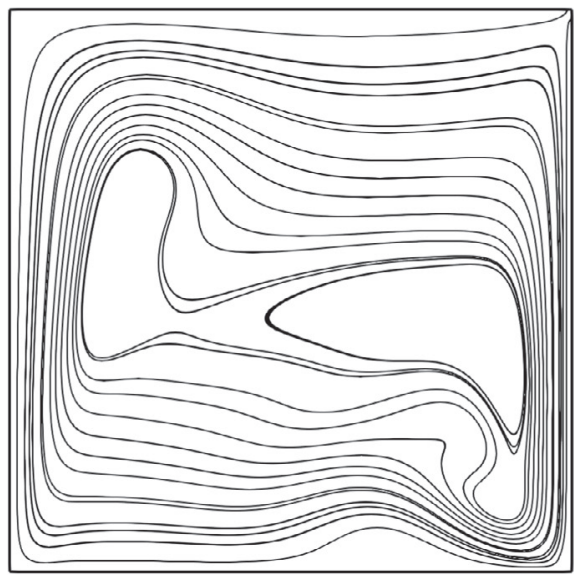

(a) Streamlines

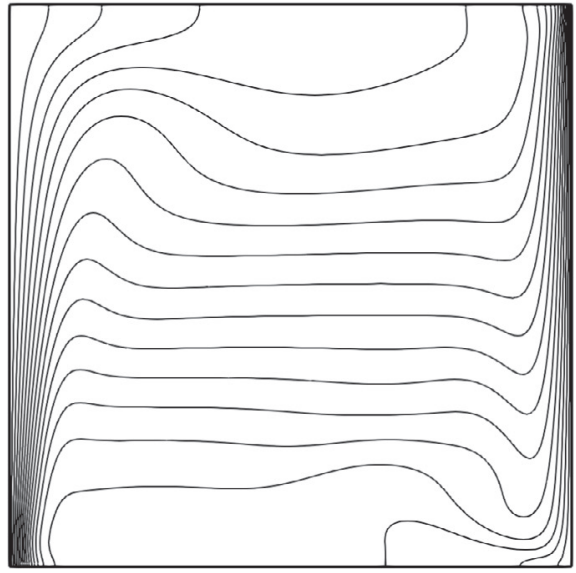

(b) Temperature contours

Fig. 5. Streamlines and temperature contours of non-Boussinesq natural convection at $R a=10^{6}$. The solution is obtained by the present LB model on grids of $200 \times 200$

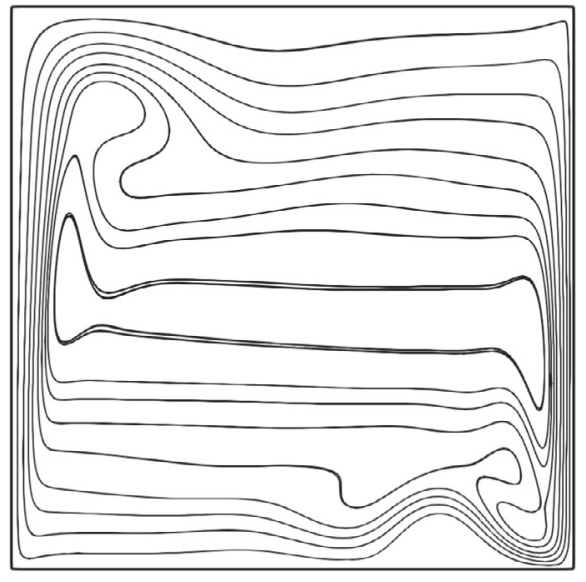

(a) Streamlines

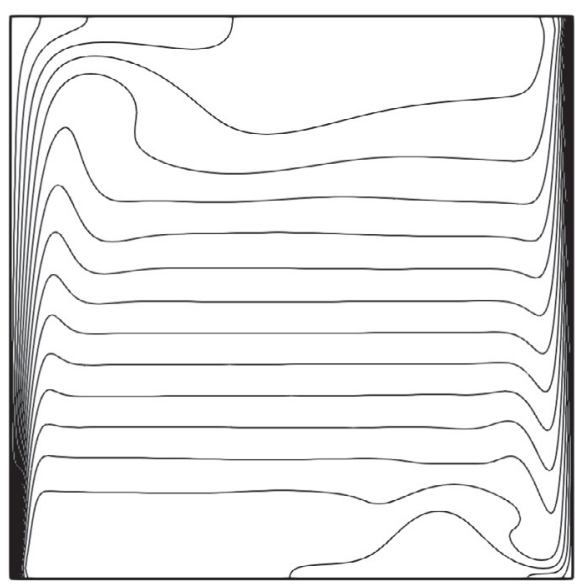

(b) Temperature contours

Fig. 6. Streamlines and temperature contours of natural convection at $R a=10^{7}$. The solution is obtained by the present LB model on grids of $400 \times 400$. 
numbers, in which the plotted results of Rayleigh numbers $R a=10^{4}, 10^{5}, 10^{6}$ are obtained on the $200 \times 200$ grid and $R a=10^{7}$ on the $400 \times 400$ grid, respectively. The slope of temperature profiles are nearly horizontal at high Rayleigh number $\left(10^{6}, 10^{7}\right)$, which indicates that the effect of convection becomes stronger with the increase of the Rayleigh number. It can be observed in Fig. 4 that the temperature profiles along the horizontal line crossing the center of the cavity are in good agreement with reference values [40]. The reference values in [40] were computed by a compressible NS solver on a $1024 \times 1024$ stretched grid. The agreement indicates that the hybrid thermal LB model with the

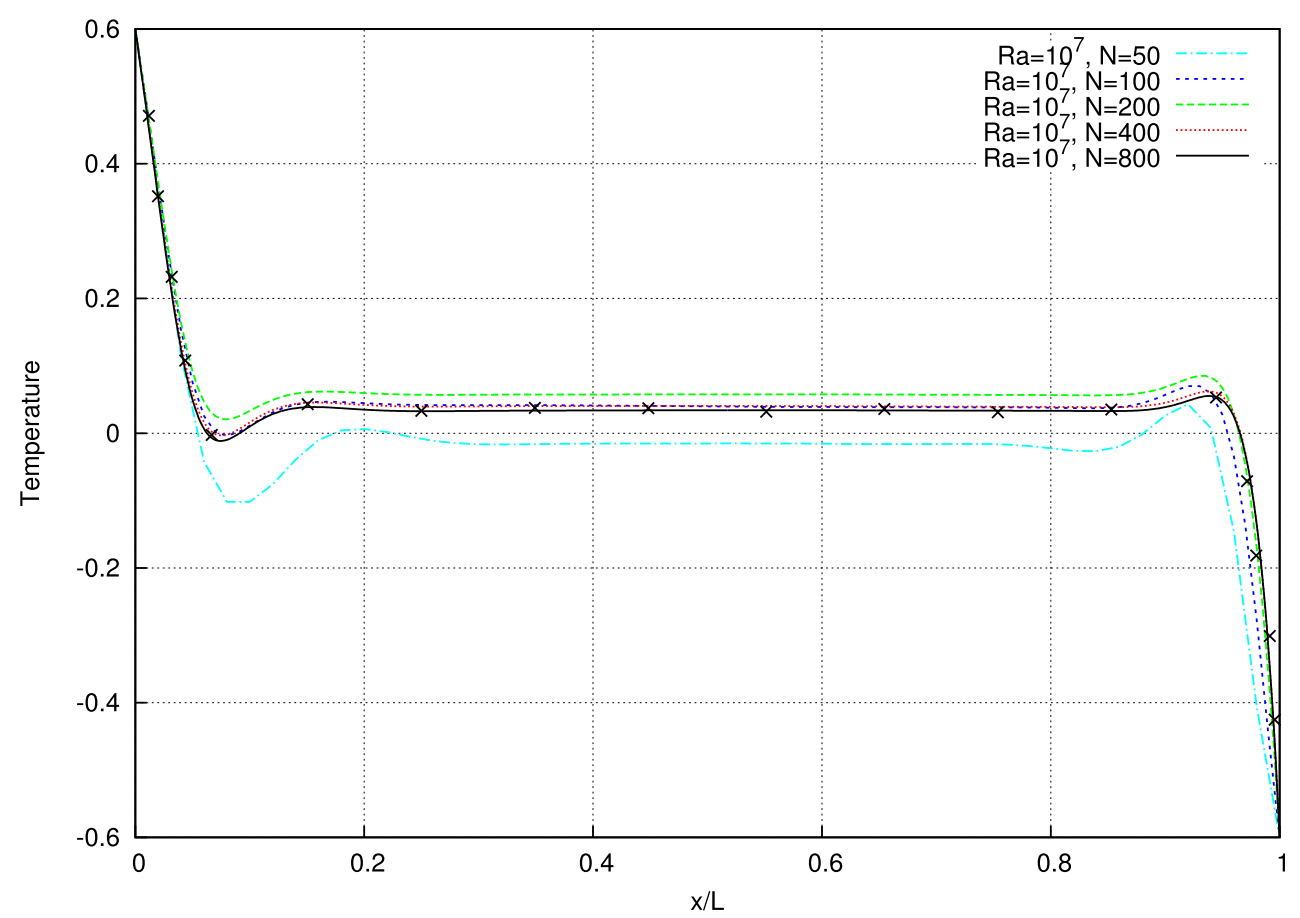

(a) Horizontal mid-line

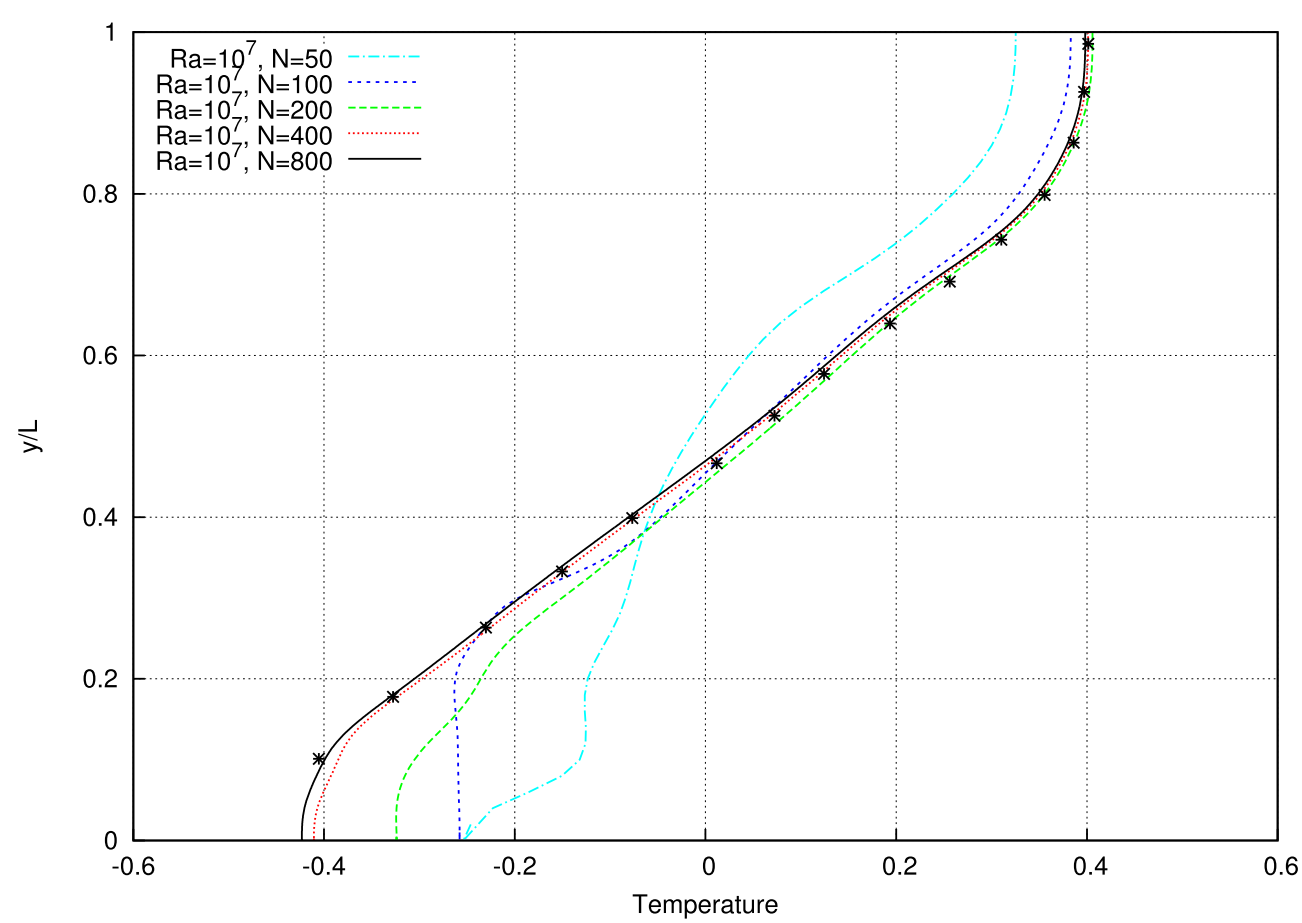

(b) Vertical mid-line

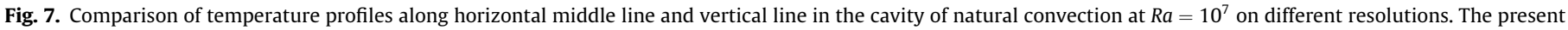
results (lines) are obtained by the hybrid LB model and the symbols represent benchmark solution in [40]. 
regularized procedure can give accurate results on non-Boussinesq natural convection in a large range of Rayleigh numbers.

Figs. 5 and 6 display the streamlines for natural convection at $R a=10^{6}, 10^{7}$. A significant difference with Boussinesq natural convection in a square cavity with constant physical properties, is that a vortex appears near the cold wall rather than near the center for the non-Boussinesq natural convection studied here. As the Rayleigh number increases from $10^{6}$ to $10^{7}$, unsteady feature of flow field increases because of the stronger buoyancy effects. The flow patterns are obviously different from those observed in Boussinesq natural convection [41], which are caused by the thermal compressibility and variable thermal properties. The temperature contours obtained by the present LB model are also shown in Figs. 5 and 6 . The isotherms become nearly horizontal at the large Rayleigh numbers. Both streamlines and temperature contours are in excellent agreement with the benchmark solutions [40]. These consistences strongly suggest that the proposed hybrid LB model exhibits good accuracy and numerical stability in simulation of natural convection with large temperature difference and high Rayleigh number.

The temperature profiles along horizontal middle line and vertical line in the cavity at $R a=10^{7}$ is plotted with comparison of different grid resolutions in Fig. 7. As can be seen from the figure, the temperature profiles obtained on fine mesh along the middle line are in excellent agreement with reference values. Moreover, the robustness of the proposed method is also validated on very coarse mesh at high Rayleigh number.

To quantitatively validate the present solutions, Table 1 reports the comparison of average Nusselt number and average pressure by the proposed LB method with the solutions given in literature [40]. The local and averaged Nusselt numbers are determined by:

$N u(y)=\left.\frac{L}{\lambda_{0}\left(T_{h}-T_{c}\right)} \lambda \frac{\partial T}{\partial x}\right|_{\text {wall }}$

$N u=\frac{1}{L} \int_{y=0}^{y=L} N u(y) d y$

The Nusselt number dramatically increases with Rayleigh number. It indicates that the effect of heat transfer between the two lateral walls becomes stronger with the increase of thermal convection. The deviations are within 3\% in all cases. This demonstrates that the good performances of the present method for this type of natural convection problems.

\subsection{Unsteady natural convection in a tall cavity}

The second test problem about natural convection deals with the thermal flow inside a heated cavity. It is somewhat similar to the previous one, but it mainly differs in two aspects: (i) the analyzed cavity is not square and is significantly larger and (ii) the flow has an unsteady character. The ratio of the height $(\mathrm{W})$ to width (L) of the cavity is $\mathrm{W} / \mathrm{L}=8 / 1$. A nondimensional parameter $\epsilon=\left(T_{h}-T_{c}\right) /\left(T_{h}+T_{c}\right)$ is introduced to represent the temperature difference in two simulated cases. Two cases are investigated and validated using the present LB method: (i) Case 1 with a small temperature difference of $\epsilon=0.1$ which gives $\Delta T=\left(T_{h}-T_{c}\right)=12 \mathrm{~K}$; (ii) Case 2 with a large temperature difference of $\epsilon=0.8$ leading to $\Delta T=960 \mathrm{~K}$. The reference temperature is $T_{0}=\left(T_{h}+T_{c}\right) / 2=600 \mathrm{~K}$ in the both cases.

The Case 1 with a small temperature difference has been extensively studied in the framework of Boussinesq approximation and was considered as the benchmark problem for unsteady flows driven by the natural convection [42-44]. It was found that for $R a=3.4 \times 10^{5}$ the flow oscillates with precisely determined amplitude and frequency.

The simulations are normalized in the same way as for natural convection under the Boussinesq approximation. The analyzed non-dimensional quantities are the temperature and time expressed as: $T^{*}=\left(T-T_{0}\right) / T, t^{*}=t / t_{r}$. The reference time is $t_{r}=L / U_{r}$ where the reference velocity is $U_{r}=g \beta L T$ and $\beta$ is the thermal expansion coefficient and equal to $1 / T$ for perfect gas.

The computations were performed on uniform grids with $100 \times 800$ for Case 1 and $200 \times 1600$ grids for Case 2 with a time step equal to $\Delta t=0.001$ and 0.000125 , respectively. Both for Case 1 and Case 2 the solutions have been initialized enforcing a zero velocity field along with a uniform temperature $T_{0}$. Both the initial condition and the boundary conditions in density distribution functions are implemented in the same way used in the previous test case.

Despite of small density changes in Case 1 with a small temperature difference, the variations of velocities and temperature exhibit a strongly unsteady behavior, which gives opportunity to assess the stability and accuracy of the proposed method for time dependent problems. Fig. 8 presents the contours of instantaneous values of temperature, horizontal velocity and vertical velocity obtained by the present LB method in Case 1 when the flows archived a fully developed state. The contours of temperature are smooth and without any spurious wiggles in Fig. 8. It is shown that this test case with small temperature difference can be well analyzed by the present model using the perfect gas law.

Table 2 summarizes the present computational results and benchmark solutions available in open literature. The computed mean values and oscillation amplitudes are denoted by an over bar and a prime, respectively. All time-mean data reported in this table are averaged over 20 periods. The Nusselt number $N u_{0}$ is computed along the hot wall and $N u_{c}$ is calculated along the center line, respectively. The difference is calculated between the present result and data in [42]. The present results are in excellent agreement with the reference data. This demonstrate the accuracy of the proposed LB model in simulation of unsteady natural convection.

The case 2 with $\epsilon=0.8$ is chosen to assess the robustness of the proposed LB method in simulation of strongly unsteady convection with large density variations $\left(\rho_{h} / \rho_{c}=9\right)$. Fig. 9 displays the contours of instantaneous values of temperature, horizontal velocity and vertical velocity obtained in Case 2 . And, Fig. 10 shows the time evolution of the non-dimensional temperature for $\epsilon=0.8$.

Table 1

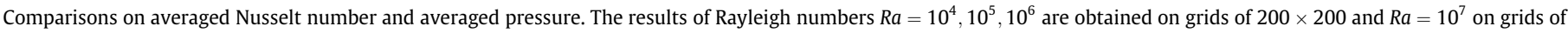
$400 \times 400$.

\begin{tabular}{|c|c|c|c|c|}
\hline \multirow[t]{2}{*}{$R a$} & \multicolumn{2}{|c|}{$\mathrm{Nu}$} & \multicolumn{2}{|c|}{$\bar{p} / p_{0}$} \\
\hline & Benchmark & Present & Benchmark & Present \\
\hline $10^{4}$ & 2.22 & 2.28 & 0.91463 & 0.92076 \\
\hline $10^{5}$ & 4.48 & 4.55 & 0.92196 & 0.92732 \\
\hline $10^{6}$ & 8.69 & 8.82 & 0.92449 & 0.93274 \\
\hline $10^{7}$ & 16.24 & 16.26 & 0.92263 & 0.92745 \\
\hline
\end{tabular}




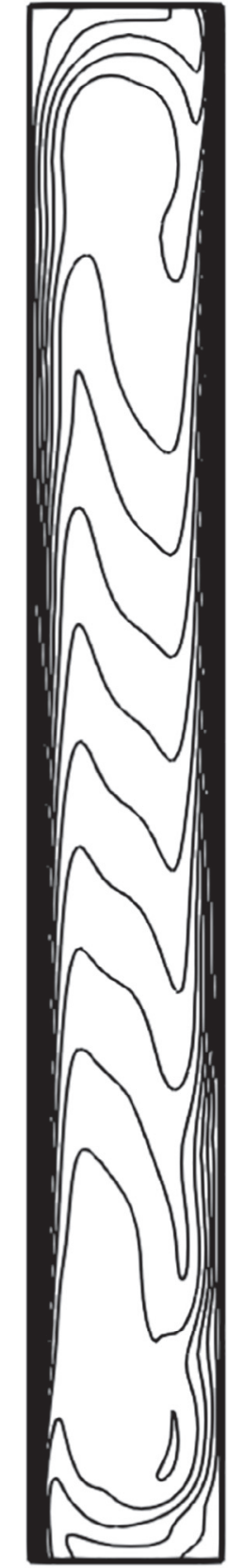

(a) Temperature

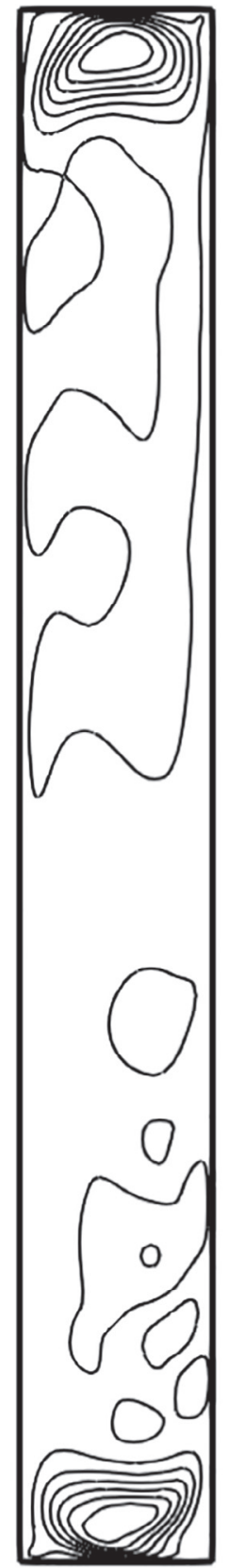

(b) Horizontal velocity

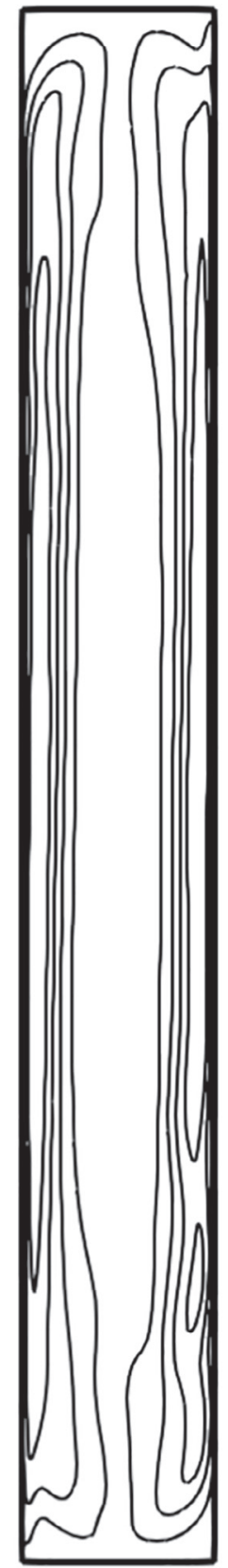

(c) Vertical velocity
Fig. 8. Contours of temperature, velocities obtained on $100 \times 800$ grid by the present LB model in natural convection with a small temperature difference of $\epsilon=0.1$.

Table 2

Comparison of Nusselt number obtained on $100 \times 800$ grid by the present hybrid LB model with benchmark solution. $N u_{0}$ is computed along the hot wall and $N u_{c}$ is calculated along the center line.

\begin{tabular}{lllll}
\hline & $\overline{N u_{0}}$ & $N u_{0}^{\prime}$ & $\overline{N u_{c}}$ & $N u_{c}^{\prime}$ \\
\hline Xin and Le Quéré & 4.57946 & 0.07094 & 4.57947 & 0.17790 \\
Gjesdal et al. & 4.57933 & 0.07010 & 4.57946 & 0.17761 \\
Present results & 4.57253 & 0.07120 & 4.57255 & 0.17924 \\
Difference (\%) & -0.151 & 0.367 & -0.151 & 0.753 \\
\hline
\end{tabular}

In the present study the variations of temperature are analyzed and compared with benchmark solutions. The time evolution of temperature at a control point was recorded during unsteady

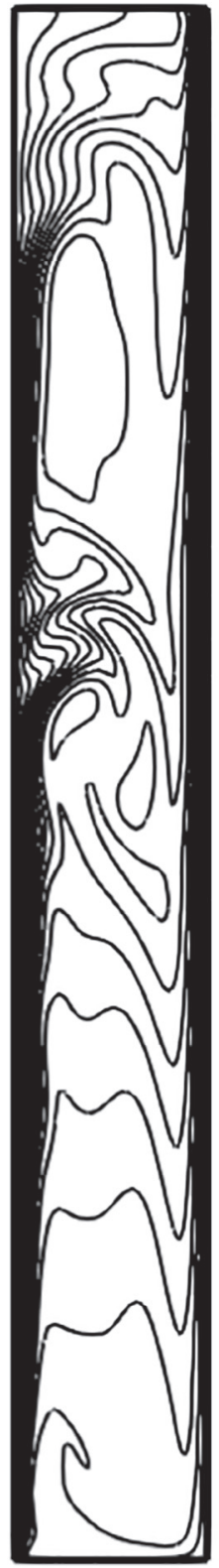

(a) Temperature

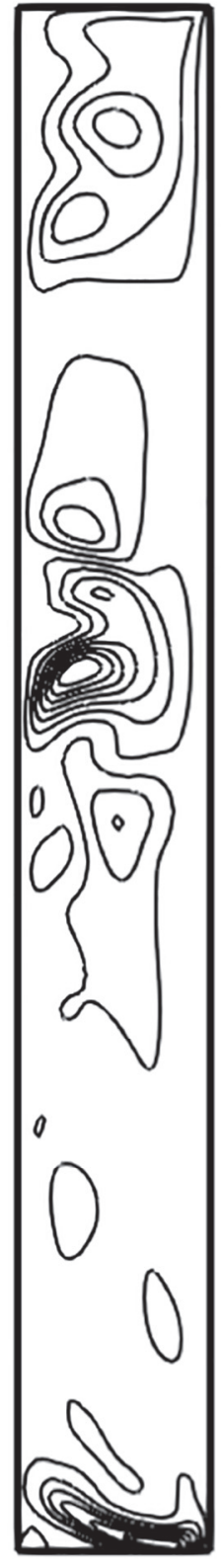

(b) Horizontal velocity

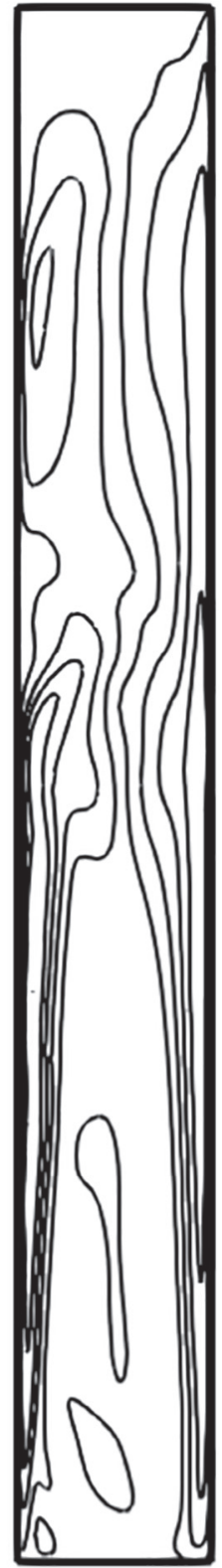

(c) Vertical velocity
Fig. 9. Contours of temperature, velocities on $200 \times 1600$ grid obtained by the present LB model for natural convection in a tall enclosure with a large temperature difference of $\epsilon=0.8$

process. The coordinates of this point was defined in [44] as $(0.181,7.37)$. On the mesh used in the present study the coordinate of grid nodes closest to that location is $(0.18,7.37)$.

Figs. 11 and 12 report the time evolution of temperature for $\epsilon=0.1$ and $\epsilon=0.8$, respectively. For natural convection with a small temperature difference $\epsilon=0.1$ the oscillations grow slowly and become regular and sinusoidal. The mean value, oscillation period and amplitude of fluctuations are precisely computed in Case 1. However, for natural convection with a large temperature difference $\epsilon=0.8$ the solution is completely different and achieve the chaotic flows.

Moreover, the accuracy of the results on unsteady evolution is quantitatively studied on natural convection with a small temperature difference, which is compared with the reference results in $[42,45]$. The compared quantities are: the mean temperature $\overline{T^{*}}=0.5 *\left(T_{\max }^{*}+T_{\min }^{*}\right)$, the amplitude $T^{* \prime}=\left(T_{\max }^{*}-T_{\min }^{*}\right)$ and the 


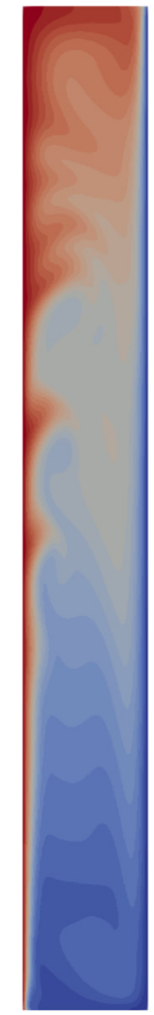

(a) time 1

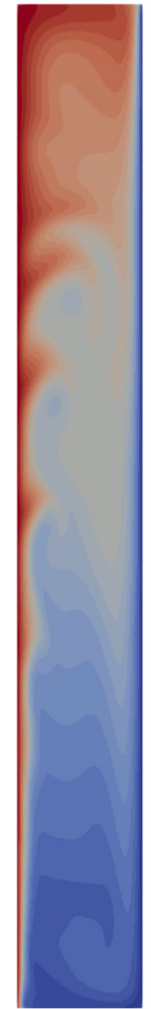

(b) time 2

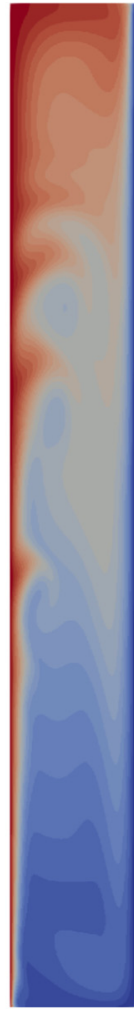

(c) time 3

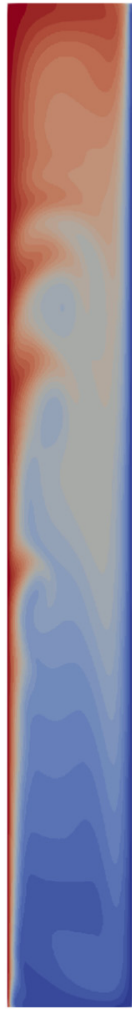

(d) time 4

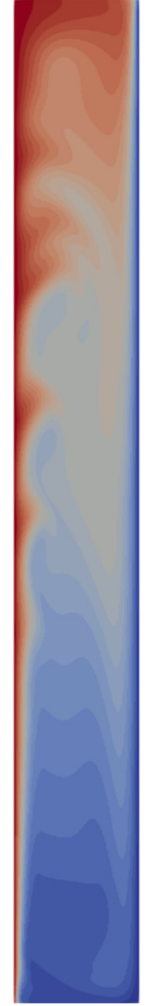

(e) time 5

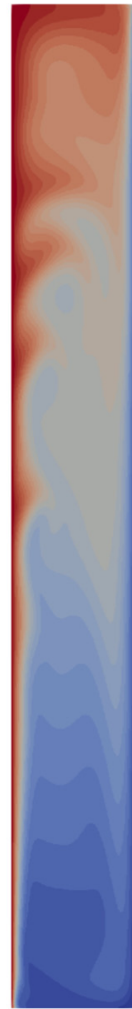

(f) time 6

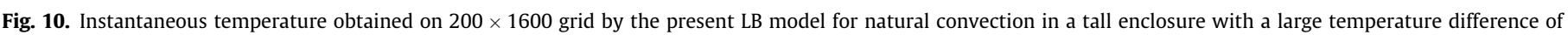
$\epsilon=0.8$.

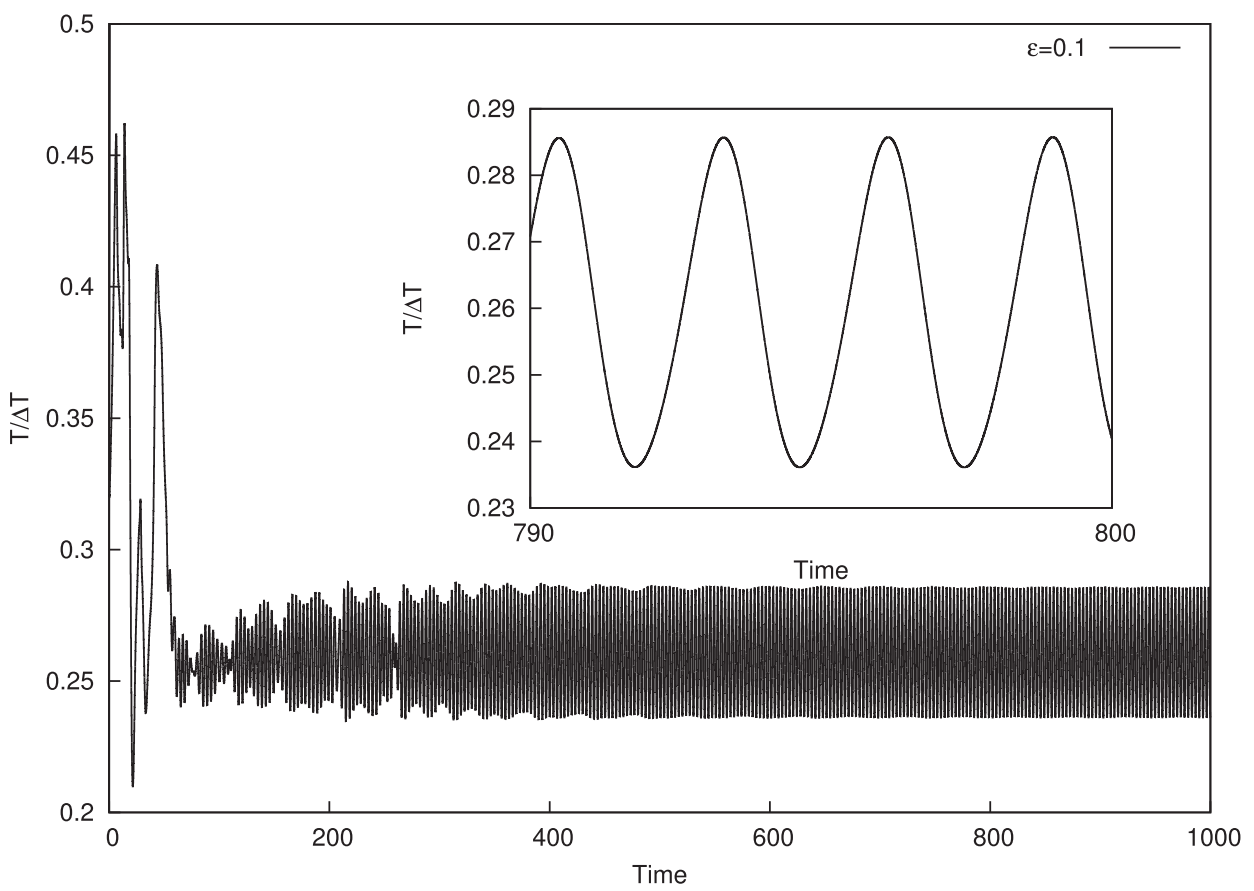

Fig. 11. Time evolution of the normalized temperature at the control point $(0.181,7.37)$ in a tall cavity with small temperature difference of $\epsilon=0.1$.

oscillation period $T_{\tau}$. Table 3 details the results taken from open literature and obtained presently in the grid closest to the control point. The ranges of values presented in Table 3 for the literature data cover the results obtained by various research groups partic- ipating in the benchmark computations. As can be seen from Table 3 , the differences between the results obtained by the present LB model are small. The proposed LB method provide accurate solutions which are closely confirmed with the reference data. 


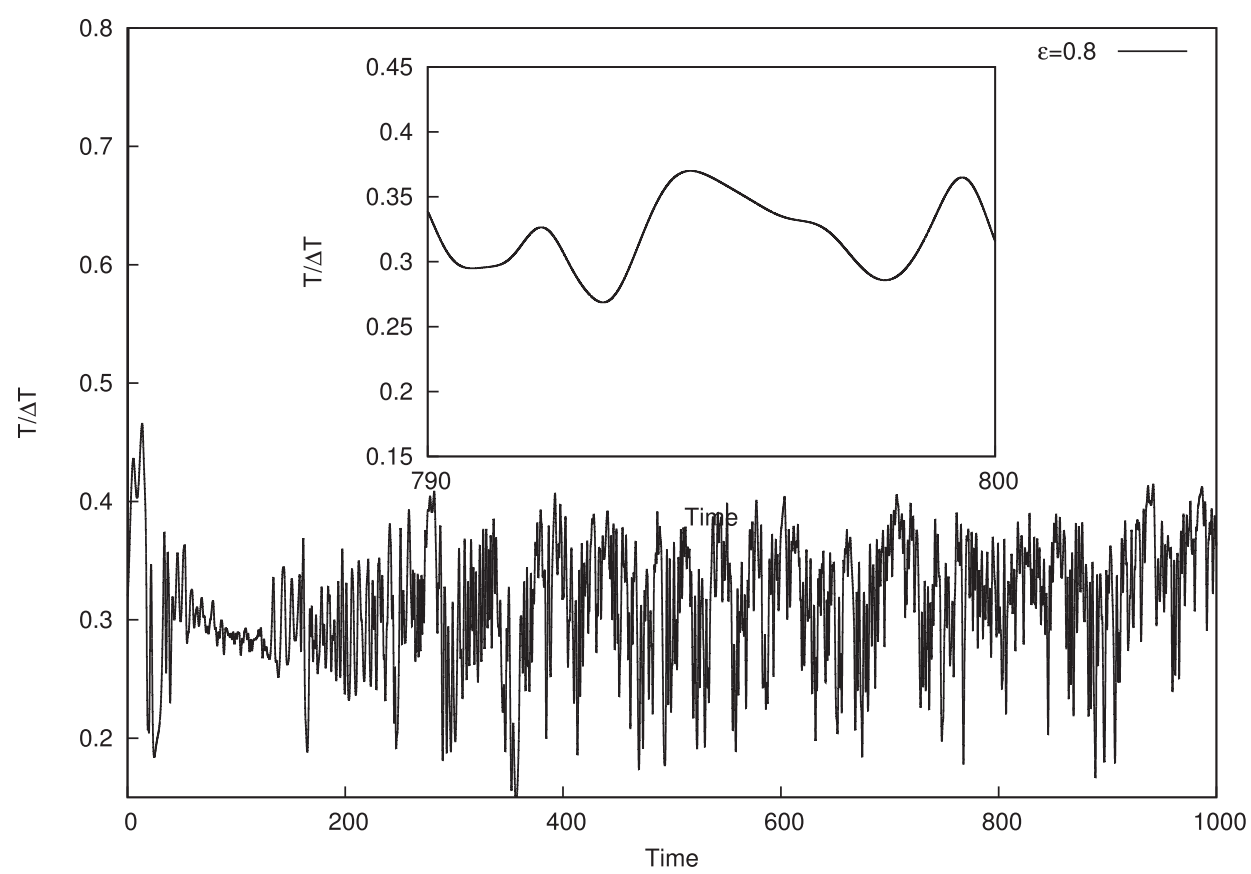

Fig. 12. Time evolution of the normalized temperature at the control point $(0.181,7.37)$ in a tall cavity with small temperature difference of $\epsilon=0.8$.

Table 3

Comparison of mean temperature, amplitude and oscillation period for natural convection in a tall cavity with a small temperature difference of $\epsilon=0.1$.

\begin{tabular}{llll}
\hline Compared quantity & $\overline{T^{*}}$ & $T^{* \prime}$ & $T_{\tau}$ \\
\hline Xin and Le Quéré & 0.265 & 0.043 & 3.412 \\
Klein et al. & 0.262 & 0.047 & 3.420 \\
Present $\epsilon=0.1$ & 0.261 & 0.047 & 3.420 \\
\hline
\end{tabular}

Based on the solution obtained for the case with a small temperature difference and the case with a large temperature difference, a concluding remark for this section is that the proposed LB method is stable and accurate in unsteady simulations for large temperature/density variations at high Rayleigh number.

\section{Conclusions}

In this paper a regularized hybrid thermal lattice Boltzmann model for natural convection with large temperature difference, variable density and high Rayleigh number has been developed. First, numerical comparisons have shown good computational efficiency and robustness of hybrid thermal LBM. The HT model with second order explicit Rung-Kutta scheme has presented faster convergence speed than a simple DDF model in simulation of natural convection under the Boussinesq approximation. Validation of hybrid thermal lattice model has been performed for nonBoussinesq natural convection with large temperature difference and high Rayleigh number. Good agreement with previously published results obtained via other numerical methods is observed on both steady and unsteady natural convection. For unsteady natural convection, the mean temperature, oscillation period and amplitude of fluctuations have been well captured in small temperature difference condition and oscillations with chaotic feature have been well observed in large temperature difference case.

\section{Conflict of interest}

The authors declared that there is no conflict of interest.

\section{Acknowledgement}

We wish to acknowledge the support by the AMIDEX project (No:ANR-11-IDEX-0001-02) funded by the "Investissements d'Avenir" French Government program and CLIMB project in the framework of the program "Investissement d'Avenir: Calcul Intensif et Simulation Numérique". This work was granted access to the HPC resources of TGCC under the allocation 2016-t20162a7679 made by GENCI. This work was partially supported by the French project CLIMB, with the financial support of BPIFrance (Project No. P3543-24000).

\section{References}

[1] S. Roller, C.-D. Munz, A low Mach number scheme based on multi-scale asymptotics, Comput. Vis. Sci. 3 (2000) 85-91.

[2] S. Succi, The Lattice Boltzmann Equation: For Fluid Dynamics and Beyond, Numerical Mathematics and Scientific Computation, Clarendon Press, 2001.

[3] L. Chen, Y.-L. Feng, C.-X. Song, L. Chen, Y.-L. He, W.-Q. Tao, Multi-scale modeling of proton exchange membrane fuel cell by coupling finite volume method and lattice Boltzmann method, Int. J. Heat Mass Transfer 63 (2013) 268-283.

[4] A. Mohamad, A. Kuzmin, A critical evaluation of force term in lattice Boltzmann method, natural convection problem, Int. J. Heat Mass Transfer 53 (5) (2010) 990-996.

[5] Y. Zhou, R. Zhang, I. Staroselsky, H. Chen, Numerical simulation of laminar and turbulent buoyancy-driven flows using a lattice Boltzmann based algorithm, Int. J. Heat Mass Transfer 47 (22) (2004) 4869-4879.

[6] X. Liu, P. Cheng, Lattice Boltzmann simulation of steady laminar film condensation on a vertical hydrophilic subcooled flat plate, Int. J. Heat Mass Transfer 62 (2013) 507-514.

[7] O. Filippova, D. Hänel, A novel lattice bgk approach for low Mach number combustion, J. Comput. Phys. 158 (2000) 139-160.

[8] L. Hung, J. Yang, A coupled lattice Boltzmann model for thermal flows, IMA J. Appl. Math. 76 (2011) 774-789.

[9] Q. Li, K. Luo, Y. He, W. Tao, Couplling lattice Boltzmann model for simulation of thermal flows on standard lattices, Phys. Rev. E 85 (2012) 016710.

[10] Y. Feng, P. Sagaut, W. Tao, A three dimensional lattice model for thermal compressible flow on standard lattices, J. Comput. Phys. 303 (2015) 514-529.

[11] P.L. Bhatnagar, E.P. Gross, M. Krook, A model for collision processes in gases. I. Small amplitude processes in charged and neutral one-component systems, Phys. Rev. 94 (1954) 511-525.

[12] P. Lallemand, L.S. Luo, Theory of the lattice Boltzmann method: acoustic and thermal properties in two and three dimensions, Phys. Rev. E 68 (2003) 036706.

[13] N.I. Prasianakis, S.S. Chikatamarla, I.V. Karlin, S. Ansumali, K. Boulouchos, Entropic lattice Boltzmann method for simulation of thermal flows, Math. Comput. Simul. 72 (2006) 179-183. 
[14] J. Latt, B. Chopard, Lattice Boltzmann method with regularized pre-collision distribution functions, Math. Comput. Simul 72 (2-6) (2006) 165-168.

[15] K. Mattila, P. Philippi, L. HegeleJr, High-order regularization in latticeBoltzmann equations, Phys. Fluids 29 (4) (2017) 046103.

[16] C. Coreixas, G. Wissocq, G. Puigt, J. Boussuge, P. Sagaut, Recursive regularization step for high-order lattice Boltzmann methods, Phys. Rev. E 96 (2017) 033306.

[17] J. Jacob, O. Malaspinas, P. Sagaut, A new hybrid recursive regularized Bhatnagar-Gross-Krook collision model for lattice-Boltzmann-method based large-eddy simulation, Phys. Fluids (in preparation).

[18] P. Lallemand, L.S. Luo, Hybrid finite-difference thermal lattice Boltzmann equation, Int. J. Mod. Phys. B 17 (2003) 41-47.

[19] A. Mezrhab, M. Bouzidi, P. Lallemand, Hybrid lattice-Boltzmann finitedifference simulation of convective flows, Comput. Fluids 33 (4) (2004) 623641.

[20] J. Tölke, A thermal model based on the lattice Boltzmann method for low Mach number compressible flows, J. Comput. Theoret. Nanosci. 3 (2006) 1-9.

[21] Q. Li, K.H. Luo, Effect of the forcing term in the pseudopotential lattice Boltzmann modeling of thermal flows, Phys. Rev. E - Stat., Nonlinear, Soft Matter Phys. 89 (5) (2014) 1-7.

[22] X. Nie, X. Shan, H. Chen, Lattice Boltzmann/Finite-difference Hybrid Simulation of Transonic Flow, AIAA Paper 139, 2009.

[23] Y. Qian, D. D'Humires, P. Lallemand, Lattice bgk models for Navier-Stokes equation, EPL (Europhys. Lett.) 17 (1992) 479-484.

[24] S.Y. Chen, G.D. Doolen, Lattice Boltzmann method for fluid flows, Annu. Rev. Fluid Mech. 30 (1998) 329-364.

[25] S. Chapman, T.G. Cowling, The Mathematical Theory of Non-Uniform Gases, Cambridge University Press, 1970.

[26] X.Y. He, S.Y. Chen, G.D. Doolen, A novel thermal model for the lattice Boltzmann method in incompressible limit, J. Comput. Phys. 146 (1998) 282 300.

[27] Z.L. Guo, C.G. Zheng, B.C. Shi, T.S. Zhao, Thermal lattice Boltzmann equation for low Mach number flows: decoupling model, Phys. Rev. E 75 (2007) 036704.

[28] Y. Saad, Iterative Methods for Sparse linear Systems, PWS Publishing, New York, 1996.

[29] D.L. Sun, Y.P. Yang, J.L. Xu, W.Q. Tao, Performance analysis of ideal algorithm combined with bi-cgstab method, Numer. Heat Transfer, Part B: Fundam. 56 (2010) 411-431.

[30] W.Q. Tao, Numerical Heat Transfer, second ed., Xian Jiaotong University Press, Xian, 2001.
[31] L.-S. Kuo, W.-P. Chou, P.-H. Chen, Effects of slip boundaries on thermal convection in $2 \mathrm{~d}$ box using lattice Boltzmann method, Int. J. Heat Mass Transfer 54 (7) (2011) 1340-1343.

[32] S. Chen, H. Liu, C. Zheng, Numerical study of turbulent double-diffusive natural convection in a square cavity by les-based lattice Boltzmann model, Int. J. Heat Mass Transfer 55 (17) (2012) 4862-4870.

[33] F. Hajabdollahi, K.N. Premnath, Central moments-based cascaded lattice Boltzmann method for thermal convective flows in three-dimensions, Int. J. Heat Mass Transfer 120 (2018) 838-850.

[34] O. Malaspinas, B. Chopard, J. Latt, General regularized boundary condition for multi-speed lattice Boltzmann models, Comput. Fluids 49 (1) (2011) 29-35.

[35] L. Li, R. Mei, J.F. Klausner, Lattice Boltzmann models for the convectiondiffusion equation: D2q5 vs d2q9, Int. J. Heat Mass Transfer 108 (2017) 41-62.

[36] X.W. Shan, X.F. Yuan, H.D. Chen, Kinetic theory representation of hydrodynamics: a way beyond the Navier-Stokes equation, J. Fluid Mech. 550 (2006) 413-441.

[37] S.P. Thampi, S. Ansumali, R. Adhikari, S. Succi, Isotropic discrete Laplacian operators from lattice hydrodynamics, J. Comput. Phys. 234 (2013) 1-7.

[38] C. Zhuo, P. Sagaut, Acoustic multipole sources for the regularized lattice Boltzmann method: comparison with multiple-relaxation-time models in the inviscid limit, Phys. Rev. E 95 (6) (2017) 063301.

[39] E. Garnier, N. Adams, P. Sagaut, Large Eddy Simulation for Compressible Flows, Scientific Computation, Springer, 2009.

[40] J. Vierendeels, B. Merci, E. Dick, Benchmark solutions for the natural convective heat transfer problem in a square cavity with large horizontal temperature differences, Int. J. Numer. Methods Heat Fluid Flow 13 (2003) 1057-1078.

[41] G. Barakos, E. Mitsoulis, D. Assimacopoulos, Natural convection flow in a square cavity revisited: laminar and turbulent models with wall functions, Int. J. Numer. Meth. Fluids 18 (1994) 695-719.

[42] S. Xin, P.L. Quéré, An extended Chebyshev pseudo-spectral benchmark for the 8: 1 differentially heated cavity, Int. J. Numer. Meth. Fluids 40 (8) (2002) 981 998.

[43] T. Gjesdal, C.E. Wasberg, B.A.P. Reif, Spectral element benchmark simulations of natural convection in two-dimensional cavities, Int. J. Numer. Methods Fluids 50 (11) (2006) 1297-1319.

[44] M.A. Christon, P.M. Gresho, S.B. Sutton, Computational predictability of timedependent natural convection flows in enclosures (including a benchmark solution), Int. J. Numer. Meth. Fluids 40 (8) (2002) 953-980.

[45] Klein, A high-order discontinuous Galerkin solver for low Mach number flows, Int. J. Numer. Methods Fluids (October 2007) (2008) 601-629. 